\title{
Executive Semantic Processing Is Underpinned by a Large-scale Neural Network: Revealing the Contribution of Left Prefrontal, Posterior Temporal, and Parietal Cortex to Controlled Retrieval and Selection Using TMS
}

\author{
Carin Whitney $^{1}$, Marie Kirk ${ }^{1}$, Jamie O'Sullivan ${ }^{1}$, \\ Matthew A. Lambon Ralph ${ }^{2}$, and Elizabeth Jefferies ${ }^{1}$
}

\begin{abstract}
To understand the meanings of words and objects, we need to have knowledge about these items themselves plus executive mechanisms that compute and manipulate semantic information in a task-appropriate way. The neural basis for semantic control remains controversial. Neuroimaging studies have focused on the role of the left inferior frontal gyrus (LIFG), whereas neuropsychological research suggests that damage to a widely distributed network elicits impairments of semantic control. There is also debate about the relationship between semantic and executive control more widely. We used TMS in healthy human volunteers to create "virtual lesions" in structures typically damaged in patients with semantic control deficits: LIFG, left posterior middle temporal gyrus (pMTG), and intraparietal sulcus (IPS). The
\end{abstract}

influence of TMS on tasks varying in semantic and nonsemantic control demands was examined for each region within this hypothesized network to gain insights into (i) their functional specialization (i.e., involvement in semantic representation, controlled retrieval, or selection) and (ii) their domain dependence (i.e., semantic or cognitive control). The results revealed that LIFG and pMTG jointly support both the controlled retrieval and selection of semantic knowledge. IPS specifically participates in semantic selection and responds to manipulations of nonsemantic control demands. These observations are consistent with a large-scale semantic control network, as predicted by lesion data, that draws on semantic-specific (LIFG and pMTG) and domain-independent executive components (IPS).

\section{INTRODUCTION}

Semantic cognition refers to the ability to assign and use the meanings of words, sounds, objects, and faces to interact with the environment. This capacity relies on both stored semantic knowledge (semantic representations) and executive control mechanisms that shape semantic activation in line with current goals and constraints (semantic control). We know a vast amount about any given concept - yet only particular aspects of our knowledge will be relevant in a given situation. For example, we know many different things about bananas, including that they are peeled before being eaten and that they can make you slip when dropped on the ground. To understand the relationship between "banana" and "slip," it is necessary to focus on a relatively obscure aspect of meaning (i.e., that a banana has a slimy texture) as opposed to more dominant aspects that are thought to be retrieved automati-

${ }^{1}$ University of York, ${ }^{2}$ University of Manchester

cally (Corbett, Jefferies, \& Lambon Ralph, 2009; Badre \& Wagner, 2007; Jefferies, Baker, Doran, \& Lambon Ralph, 2007; Jefferies \& Lambon Ralph, 2006; Badre, Poldrack, Pare-Blagoev, Insler, \& Wagner, 2005; Wagner, Pare-Blagoev, Clark, \& Poldrack, 2001; Thompson-Schill, D'Esposito, Aguirre, \& Farah, 1997). Semantic control processes therefore are a principle component of semantic cognition and interact with stored semantic knowledge during meaning retrieval.

Neuroimaging and neuropsychological research investigating the brain mechanisms underpinning semantic control have highlighted the importance of the left inferior frontal gyrus (LIFG). LIFG activation typically increases when weak or unusual relationships need to be identified in an association task, subordinate meanings of an ambiguous word need to be accessed, or the number of response options is increased, strengthening competition among potential target items in the semantic network (e.g., Badre et al., 2005; Noppeney, Phillips, \& Price, 2004; Wagner et al., 2001; Thompson-Schill et al., 1997). Moreover, LIFG 
lesions in patients with stroke aphasia (SA) produce impairments on similar tasks, establishing a causal relationship between LIFG and semantic control deficits (Noonan, Jefferies, Corbett, \& Lambon Ralph, 2010; Corbett, Jefferies, Ehsan, \& Lambon Ralph, 2009; Corbett, Jefferies, \& Lambon Ralph, 2009; Novick, Kan, Trueswell, \& Thompson-Schill, 2009; Soni et al., 2009; Jefferies, Patterson, \& Lambon Ralph, 2008). These studies show that individuals with SA have difficulty selecting appropriate concepts in the face of potent distracters-for example, they have difficulty retrieving the subordinate meanings of ambiguous words and struggle to reject highly associated distractor words in synonym judgment. Moreover, they profit from cues provided to reduce the requirement for internally generated semantic control (e.g., /t/ to cue "tiger" during picture naming), demonstrating that semantic knowledge itself is preserved in the face of impaired semantic control.

Contradictory conclusions, however, have been drawn about the broader semantic control network, extending beyond LIFG. One source of controversy follows from the fact that semantic control deficits are associated with lesions to both LIFG and/or temporo-parietal cortex (Novick et al., 2009; Jefferies \& Lambon Ralph, 2006). Although this suggests a large-scale distributed network, the patients' lesions are typically extensive, encompassing potentially separable sites - for example, posterior middle temporal gyrus (pMTG; BA 21/BA 37) and parietal areas (e.g., intraparietal sulcus [IPS] and BA 39/BA 40; Noonan et al., 2010). Moreover, individuals rarely have specific behavioral impairments but are likely to have damage to more than one cognitive function. Performance is impaired on a variety of standard assessments probing attentional/executive skills outside the verbal domain (e.g., deficits occurred on the WCST and the Brixton Spatial Rule Attainment task; Novick et al., 2009; Jefferies \& Lambon Ralph, 2006), suggesting that some of the regions affected in SA might serve a domain-independent control function.

Neuroimaging studies offer higher spatial resolution, but the interpretation of brain activation remains ambiguous. For example, in most fMRI investigations, high semantic control demands are confounded with the number of potential target concepts; thus, these conditions might generate greater activation in the semantic store (cf. Snijders et al., 2009; Gennari, MacDonald, Postle, \& Seidenberg, 2007; Noppeney et al., 2004). In line with this, pMTG has been described as a store for semantic knowledge that receives modulatory signals from prefrontal cortex during the process of meaning retrieval (Binder, Desai, Graves, \& Conant, 2009; Hickok \& Poeppel, 2004; Indefrey \& Levelt, 2004; Gold \& Buckner, 2002). This interpretation is in stark contrast to the neuropsychological profile of semantically impaired patients with SA: Irrespective of whether they have left prefrontal or posterior damage (encompassing pMTG plus other posterior temporal and inferior parietal areas), SA patients are able to retrieve conceptual knowledge when the control demands of semantic tasks are reduced, suggesting that this region does not constitute a key semantic store (e.g., Jefferies \& Lambon Ralph, 2006).

Although pMTG is specifically implicated in semantic processing, the left IPS, in contrast, does not appear to be specific for semantic operations, as neural activity in this area is modulated by a variety of cognitive tasks that probe executive or attention processes, including spatial orientation, tone discrimination, finger movement sequencing, and categorization of faces, as well as tasks using semantic stimuli (Hedden \& Gabrieli, 2010; Ciaramelli, Grady, \& Moscovitch, 2008; Collette, Hogge, Salmon, \& Van der Linden, 2006; Duncan, 2006; Wager, Jonides, \& Reading, 2004). Because of this domain independence, IPS has been implicated in the "multiple-demand" (MD) network alongside medial and dorsal prefrontal structures (Duncan, 2006, 2010; Cristescu, Devlin, \& Nobre, 2006; Duncan \& Owen, 2000; Owen, Schneider, \& Duncan, 2000). Some of the impairments seen after temporo-parietal infarcts in SA patients might, thus, be a consequence of damage to a MD region (i.e., IPS) rather than lesions to a semantic-specific control area. We propose that semantic control draws on both areas that are selectively engaged during tasks that require manipulation of conceptual knowledge (i.e., LIFG and pMTG) plus regions that serve a more general purpose (i.e., the allocation of attention; IPS). However, patient studies cannot investigate the separate roles of pMTG and IPS in these aspects of semantic cognition and attention, because SA patients with posterior lesions typically have damage to both of these structures.

The aim of this study was to explore the contribution of LIFG, pMTG, and IPS to control processes focusing on (i) semantic knowledge and (ii) perceptual decisions with low conceptual content (i.e., "nonsemantic" control). To resolve some of the ambiguities arising from previous research, we utilized TMS, which induces a focal and transient disruption of neural processing when applied repetitively (i.e., a "virtual lesion"; Pascual-Leone, Walsh, \& Rothwell, 2000; Walsh \& Cowey, 2000; Walsh \& Rushworth, 1999; Pascual-Leone et al., 1998). Although this technique has been successfully used to complement neuroimaging and neuropsychological studies of semantic processing (Lambon Ralph, Pobric, \& Jefferies, 2009; Devlin \& Watkins, 2007; Pobric, Jefferies, \& Lambon Ralph, 2007; Devlin, Matthews, \& Rushworth, 2003; Wassermann et al., 1999), there are next-to-no TMS studies specifically focused on semantic control. The TMS method enabled us to explore the individual contribution of relatively small cortical fields, which cannot be easily separated in studies of neuropsychological cases (i.e., sites such as pMTG and inferior parietal lobe). Furthermore, TMS is an interference technique and can establish whether stimulated regions play an essential role in particular functions, unlike fMRI. For example, pMTG shows activation during highcontrol semantic tasks in fMRI, but this activation may not be necessary for semantic control-instead, it may reflect the retrieval of a greater number of concepts in these conditions. However, if TMS to pMTG disrupts semantic 
control, this would be powerful evidence that this region does play a critical role.

To investigate the specific function of each of the target areas, we employed two manipulations of semantic control. There were two tasks with high semantic control demands, which were compared with a condition involving minimal control requirements (see Figure 1). Both control manipulations have been shown to produce greater activation in LIFG, plus pMTG and/or IPS (Badre et al., 2005; Thompson-Schill et al., 1997; Wagner et al., 2001), making these tasks ideal to assess the extent of the semantic control network outside LIFG. In the first of the high-control tasks, participants had to choose a target concept that was weakly related to the cue word, as opposed to strongly related (e.g., salt-grain vs. salt-pepper). In these situations, additional executive resources are required to retrieve the target concept from memory (i.e., "controlled semantic retrieval"), because the cue will not effectively activate the target via spreading activation in the semantic network (Wagner et al., 2001; Masson, 1991; Collins \& Loftus, 1975). In the other task, participants were asked to attend to specific, typically less salient features of word meaning (e.g., color: salt-dove; both concepts are white) while ignoring strong but task-irrelevant semantic associations at the same time (e.g., pepper was also presented as a distractor; see Figure 1). This semantic control process differed from controlled semantic retrieval in two ways: First, participants had to select a particular semantic feature, which was task relevant over competing prepotent but irrelevant information (i.e., "semantic selection"; Badre et al., 2005; ThompsonSchill et al., 1997). Second, the task required a strategic top-down approach for meaning recovery, evoked by specific task instructions (i.e., "associate: color!"). Theories that differentiate between strategic (top-down) and stimulusdriven (bottom-up) forms of attention have linked IPS to conditions where prior information biases task performance, for example, when cues indicate the position of the target on the screen versus no cues (Ciaramelli et al., 2008). In the feature selection task, the instruction biased participants' attention toward a specific semantic dimension and away from strong associations, which acted as distractors. Hence, we predicted that TMS over IPS would disrupt the feature selection task, but not the retrieval of weak associations, for which no biases/cues were necessary. In contrast, TMS to pMTG was expected to disrupt both tasks tapping semantic control, because fMRI has revealed activation increases during both conditions (i.e., during attention to specific features and for weakly related cue-target pairs; Wagner et al., 2001; Thompson-Schill et al., 1997).

To explore the roles of LIFG, pMTG, and IPS in control functions beyond the semantic domain, a perceptual matching task was designed using compound letters (e.g., an A made of small Bs), with two different levels of executive/attentive demand (Navon, 1977). Task demands were higher when participants had to match a cue letter to the local elements of a compound letter as opposed to its overall shape (see Figure 1). This process required inhibition
Figure 1. Example trials for the semantic tasks and the Navon tasks. Participants had to select the target word that was either strongly related to the cue shown above (high relatedness), weakly related (low relatedness), or unrelated but similar to the cue in one of the following semantic dimensions: color, shape, size, or texture (feature selection). In the Navon tasks, participants had to choose the target compound letter that resembled the cue letter either in its global shape (global Navon) or in its local, smaller elements (local Navon). Target items are underlined, and compound letters are enlarged for illustration purposes.

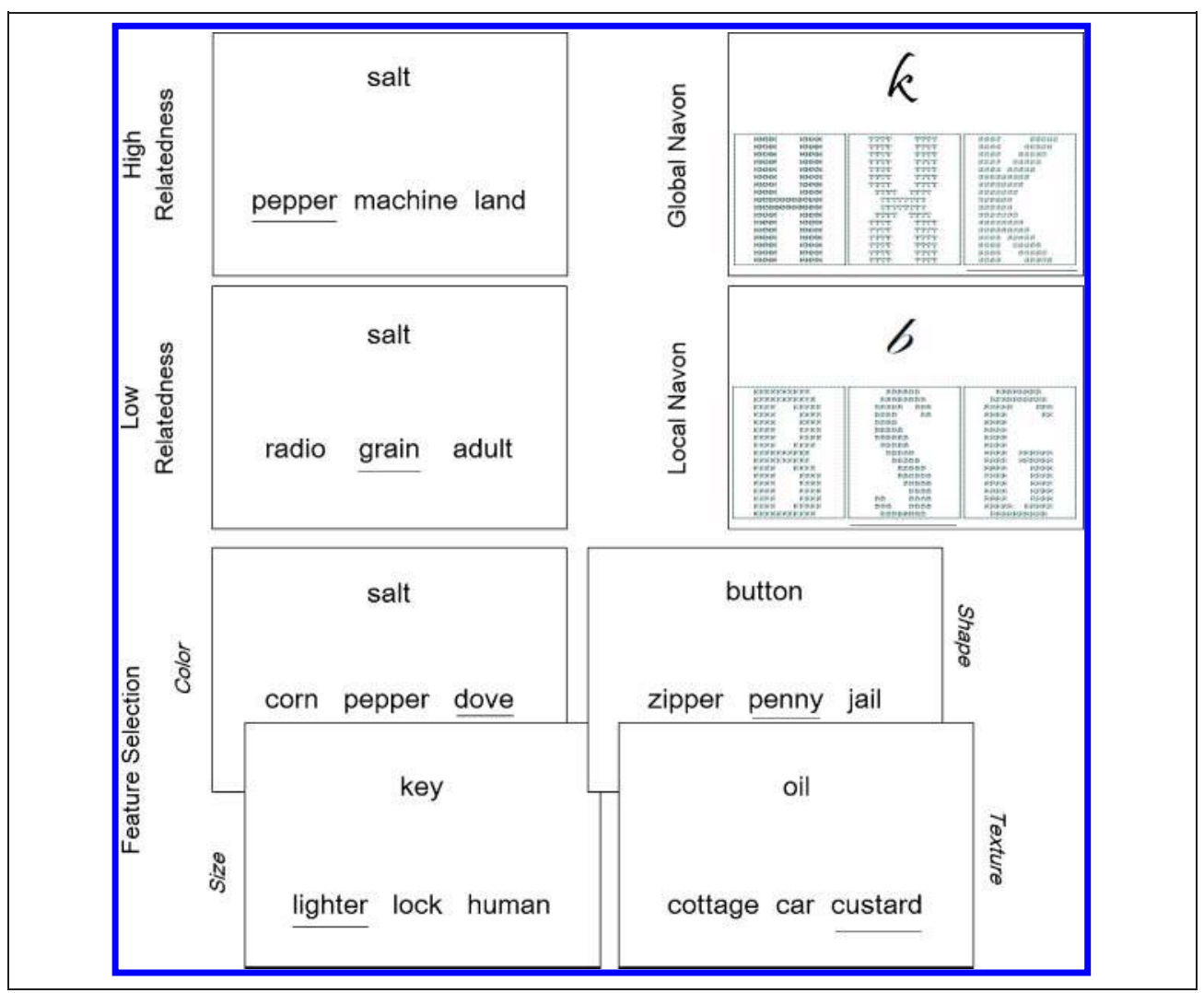


of the visually dominant global shape of the compound letter plus top-down attention directed toward selective, subordinate features of the stimulus. As such, the condition was comparable to the semantic feature selection task with the exception that participants had to orient toward spatial/perceptual properties as opposed to semantic features of the stimulus (e.g., color). fMRI and TMS studies have shown parietal cortex to be crucial for the Navon task (Hedden \& Gabrieli, 2010; Billington, Baron-Cohen, \& Bor, 2008; Mevorach, Shalev, Allen, \& Humphreys, 2008; Mevorach, Humphreys, \& Shalev, 2005), which is consistent with the view that this region serves an MD function not limited to semantic processes. The Navon task was, therefore, used to probe the domain independence of potential semantic control regions. We predicted that TMS over IPS would impair performance on the Navon taskbecause of its function in the MD network mediating topdown control-but that no TMS effects would emerge after stimulation of LIFG nor pMTG because of the low semantic content of the Navon stimuli.

\section{METHODS}

\section{Participants}

Sixteen right-handed native English speakers from the University of York participated in this study (eight women; mean age $=22.25$ years, $S D=3.55$ ). All subjects passed TMS and MRI safety screening (Wassermann, 1998), were free of medication, and did not have any personal or family history of neurological or psychiatric illness. Participants had normal or corrected-to-normal vision and gave informed consent before the beginning of the study. A reimbursement of $£ 40$ was paid for participation. The study was approved by the local ethics committee.

\section{Tasks}

Three semantic judgment tasks requiring different levels of executive semantic demand were employed (i.e., judgments involving high relatedness, low relatedness, and feature selection; see Figure 1). In each task, a cue word appeared above a row of three potential target words. Participants were asked to decide which target was related to the cue by pressing one of three buttons with their right hand, corresponding to the position of the response item (left, middle, and right).

In the high relatedness task, the target was strongly related to the cue and appeared with two unrelated distractor items (salt-pepper, machine, land). Semantic control demands were minimal because target retrieval benefitted from automatic spreading activation (Masson, 1991; Neely, 1990; Collins \& Loftus, 1975). In the low relatedness condition, cue-target associations were weak (salt-grain, radio, adult) and consequently target retrieval required additional executive resources that helped to direct the search and recovery of the relevant item, that is, "controlled se- mantic retrieval" (Badre et al., 2005; Wagner et al., 2001). In the feature selection task, the target shared a particular semantic dimension (color, size, shape, or texture) with the cue (e.g., color: salt-dove, corn, pepper). The target appeared together with a strong semantic associate and an unrelated distractor. Target retrieval required the explicit selection of the appropriate semantic feature (e.g., white) and the suppression of the dominant but irrelevant associate (pepper; "semantic selection"; Badre et al., 2005; Thompson-Schill et al., 1997). Participants' attention was biased toward a particular semantic feature before stimulus presentation (via an instruction slide, for example, "associate: color!"). Therefore, the feature selection task and the low relatedness task tapped two different forms of semantic control.

Nonsemantic control tasks were constructed from the global-local Navon letter-matching task (Navon, 1977). We produced easy and difficult versions of this task to establish whether rTMS effects over LIFG/pMTG/IPS remained specific to the semantic domain when control demands were increased. In both Navon conditions, a cue letter appeared above three larger compound letters, which were composed of smaller letters (e.g., a large letter $S$ made of small Bs; see Figure 1). In the easy condition, participants were asked to decide which compound letter matched the cue in global shape, irrespective of the letters that appeared as smaller elements inside the compound. Cognitive control demands were expected to be minimal in these trials because the global shape is visually dominant over local features (Navon, 1977). Moreover, neither distractor was related to the cue, that is, neither global shape nor local letter features matched the cue in this condition (see Figure 1). In contrast, the more difficult local Navon task required participants to match the cue letter to the local elements of one of the compounds, hence, to disregard the dominant, global shape of the stimuli. Cognitive control demands were further increased by presenting a compound letter whose global shape was identical to the cue, thus generating a strong task-irrelevant competitor (see Figure 1). The local Navon task was therefore comparable to the feature selection condition, because both required top-down control processes to direct attention away from dominant targets and toward selective, subordinate attributes of the stimulus.

\section{Design and Procedure}

A within-subject factorial design was used, with stimulation SITE (LIFG, pMTG, IPS), TMS (stimulation vs. no stimulation), and TASK (three semantic tasks, two Navon tasks) as within-subject factors. Each site was stimulated on a different day, with test sessions separated by at least 1 week. The sequence of stimulation site was counterbalanced across sessions. Furthermore, each session included recordings of task performance immediately after TMS and without any TMS intervention ("baseline" performance) to identify the influence of TMS on cognitive behavior 
(TMS effect). Baseline performance was measured either before TMS intervention or $30 \mathrm{~min}$ after TMS offset by which time no TMS effect remains (Pobric, Jefferies, \& Lambon Ralph, 2010; Lambon Ralph et al., 2009; Pobric, Lambon Ralph, \& Jefferies, 2009; Pobric et al., 2007). The order of baseline assessment was counterbalanced across sessions.

The six experimental runs - that is, baseline and postTMS performance for each stimulation site (LIFG, pMTG, IPS)_lasted about $6 \mathrm{~min}$ each $(M=5.93 \mathrm{~min}, S D=0.49)$ and included 30 trials per condition. Two miniblocks of 15 consecutive trials were created for each condition and presented in a pseudorandomized order to control for effects that relate to the fading of the TMS effect over time.

At the beginning of each block, an instruction slide was shown, followed by a fixation cross for $500 \mathrm{msec}$ in the center of the screen. This was replaced by the first experimental trial, which displayed the cue above three response options for a maximum of $5 \mathrm{sec}$ (Figure 1). As soon as a response was made, the fixation cross appeared again, followed by the next trial. A computer running E-prime (Psychology Software Tools) was used to present the stimuli and record the responses.

\section{Stimuli}

Each of the semantic conditions consisted of 180 cuetarget-distractor trials. The trials were arranged into six matched sets of 30 trials each, used for each experimental run, and then split into miniblocks of 15 trials, which were equated for word length, frequency, and cue-target association strength. Stimuli were based on Badre et al. (2005) but restricted to nouns only, and some trials were amended to make them suitable for U.K. participants. Words in all three conditions were matched in length in letters (low relatedness: $M=5.1, S D=1.4$; high relatedness: $M=5.2, S D=1.8$; feature selection: $M=5.5, S D=$ 1.8) and frequency (Kucera \& Francis, 1967; low relatedness: $M=54.3, S D=105.1$; high relatedness: $M=48.1$, $S D=90.4$; feature selection: $M=44.5, S D=103.6)$.

The high and low relatedness tasks were arranged such that the same cue word was matched with a high or low semantic associate, using several sets of association norms (Moss \& Older, 1996; Postman \& Keppel, 1970). Association strength was defined as the proportion of subjects that named the target in response to the cue in free association. Each cue word was also paired with two unrelated distractor items, for which no entry in the association norms was found (e.g., high: salt-pepper, machine, land; low: salt-grain, radio, adult). The mean association strength for high and low related cue-target pairs differed significantly (paired $t$ test: high $=0.24, S D=0.18$; low $=0.04, S D=$ $0.10 ; t=15.00 ; p<.001)$. For the feature selection task, cue words were paired with an unrelated target word that shared a particular semantic dimension with the cue (i.e., color, shape, size, or texture), a strong semantic associate (mean association strength $=0.22, S D=0.76$ ) and a new, unrelated distractor noun (e.g., color: salt-dove, pepper, cone). Sixteen percent of the cues and strong semantic associates were taken from the cue-target pairs in the high relatedness condition and paired with a new target and a new unrelated noun. Twelve percent of the trials in the feature selection task needed to be repeated but never within the same experimental session.

For the nonsemantic control conditions, 180 trials of the global and local version of the Navon task were constructed and, again, broken down into sets of 15 trials. Navon stimuli were taken from Hills and Lewis (2007). These depicted 21 upper-case letters (excluding M, N, Q, $\mathrm{V}, \mathrm{W})$ composed of smaller capital letters with a different identity (e.g., an A made out of small Bs). The local elements (width $\times$ length: $7 \times 7$ pixels) were arranged densely in the shape of the larger compound letter $(69 \times$ 166 pixels), with no gap in between. There were between 3 and 10 different versions of each of the 21 upper-case letters (made up of different local letters), yielding a total of 125 unique compound letters. The cues in the Navon task were 21 lower-case letters that matched the local elements or global shape of the target compound letter. To increase the number of trials and to delay response times, varying script fonts were used (Blackadder, Curlz MT, Bradleyhand, Edwardian Script, and Pristina), yielding 74 individual cue letters. No cue letters were repeated in a single experimental run.

\section{TMS Protocol}

A standard off-line "virtual lesion" rTMS protocol was used, which was compatible with established TMS safety guidelines (Rossi, Hallett, Rossini, \& Pascual-Leone, 2009; Wassermann, 1998). In the absence of any behavioral task, repetitive trains of TMS were delivered at $1 \mathrm{~Hz}$ to the target brain area for $10 \mathrm{~min}$. This repetitive stimulation results in a temporary and reversible disruption of neural processing in the underlying tissue, which interferes with tasks that rely on the stimulated area (Lambon Ralph et al., 2009; Pobric et al., 2007, 2009; Pascual-Leone et al., 1998). The resulting behavioral deficits are typically reflected in a delay in response times rather than a decline in accuracy (Devlin et al., 2003; Pascual-Leone et al., 2000; Walsh \& Cowey, 2000).

A 50-mm figure-of-eight coil, attached to a Magstim Rapid2 stimulator, was used for the repetitive delivery of magnetic pulses. The center of the coil was aligned to the point that marked the stimulation site on a tight-fitting elastic cap, worn by the participant. The coil was held firmly against the scalp throughout stimulation. Stimulation intensity was determined before each rTMS administration as $120 \%$ of active motor threshold (MT). MT was identified as the lowest intensity that produced a visible muscle twitch in the tense right hand when intensity was gradually decreased during single-pulse stimulation of left motor cortex. Intensity threshold was set to a maximum of $65 \%$ of stimulator output (mean intensity $=62.40 \%, S D=3.20$ ). 
Coil orientation was manipulated to minimize participants' discomfort during rTMS (particularly over LIFG), as previous research found behavioral effects were insensitive to the orientation of the coil (Niyazov, Butler, Kadah, Epstein, \& Hu, 2005). Also, six participants received a slightly lower intensity for rTMS over this site, ranging from $109 \%$ to $116 \%$ of individual MT $(M=113 \%)$. Despite these adaptations, LIFG stimulation yielded the strongest performance deficits, which were comparable in size to the interference observed in previous rTMS studies that used the same stimulation protocol and similar semantic tasks (e.g., Lambon Ralph et al., 2009; Pobric et al., 2007). Moreover, differences in sensory experiences across stimulation sites (e.g., in general discomfort, noise, or muscle twitches, which were highest during LIFG stimulation) cannot account for the TMS effects because (i) performance was always measured in the absence of any ongoing brain stimulation and (ii) various control tasks were used to detect any task-independent consequences of TMS (i.e., the high relatedness and Navon tasks, which were meant to have no effect after LIFG stimulation).

\section{Localization of Stimulation Sites}

Structural T1-weighted MRI scans of each participant were used to guide coil positioning using the Ascension Minibird magnetic tracking device and MRIreg software. Five anatomical landmarks (tip and bridge of the nose, left and right tragus and vertex) were identified to coregister the participant's head, stabilized on a chin rest, with the MRI image on the screen.

Our stimulation sites were derived from peak activations identified in fMRI studies that employed the same stimulus set and/or the same tasks (i.e., low and high relatedness, feature selection; Badre et al., 2005; Wagner et al., 2001; Thompson-Schill et al., 1997). One of these studies reported activation in all three target sites during increased executive semantic demands (Badre et al., 2005). Coordinates were transformed into individual subject space using the transformation matrix from the "segment" function implemented in SPM5, after the origin of each individual image was realigned to the anterior commissure. Visual inspection ensured that coordinates referred to the target areas by making reference to anatomical landmarks (Figure 2).

Activation peaks within LIFG, observed during previous fMRI studies of semantic control, were typically large and widely distributed, comprising both anterior and posterior segments of this structure. We used the Montreal Neurological Institute (MNI) coordinates for LIFG (-54 21 12) from Badre et al. (2005). This area (BA 44/45) in the pars triangularis (cf. Keller, Crow, Foundas, Amunts, \& Roberts, 2009) has been found to be sensitive to several executive
Figure 2. Stimulation sites. rTMS was delivered to the pars triangularis of LIFG, pMTG, and IPS. Images on the right include probability maps, which were available for target regions in BA 44 and BA 45 and the superior (SPL) and inferior parietal lobe (IPL). Stimulation sites are displayed on axial and saggital slices in MNI space, with reference to $y$ and $x$ coordinates, respectively. orange $=$ pars triangularis, yellow $=$ inferior and superior temporal sulcus, purple $=$ Sylvian fissure, blue = IPS

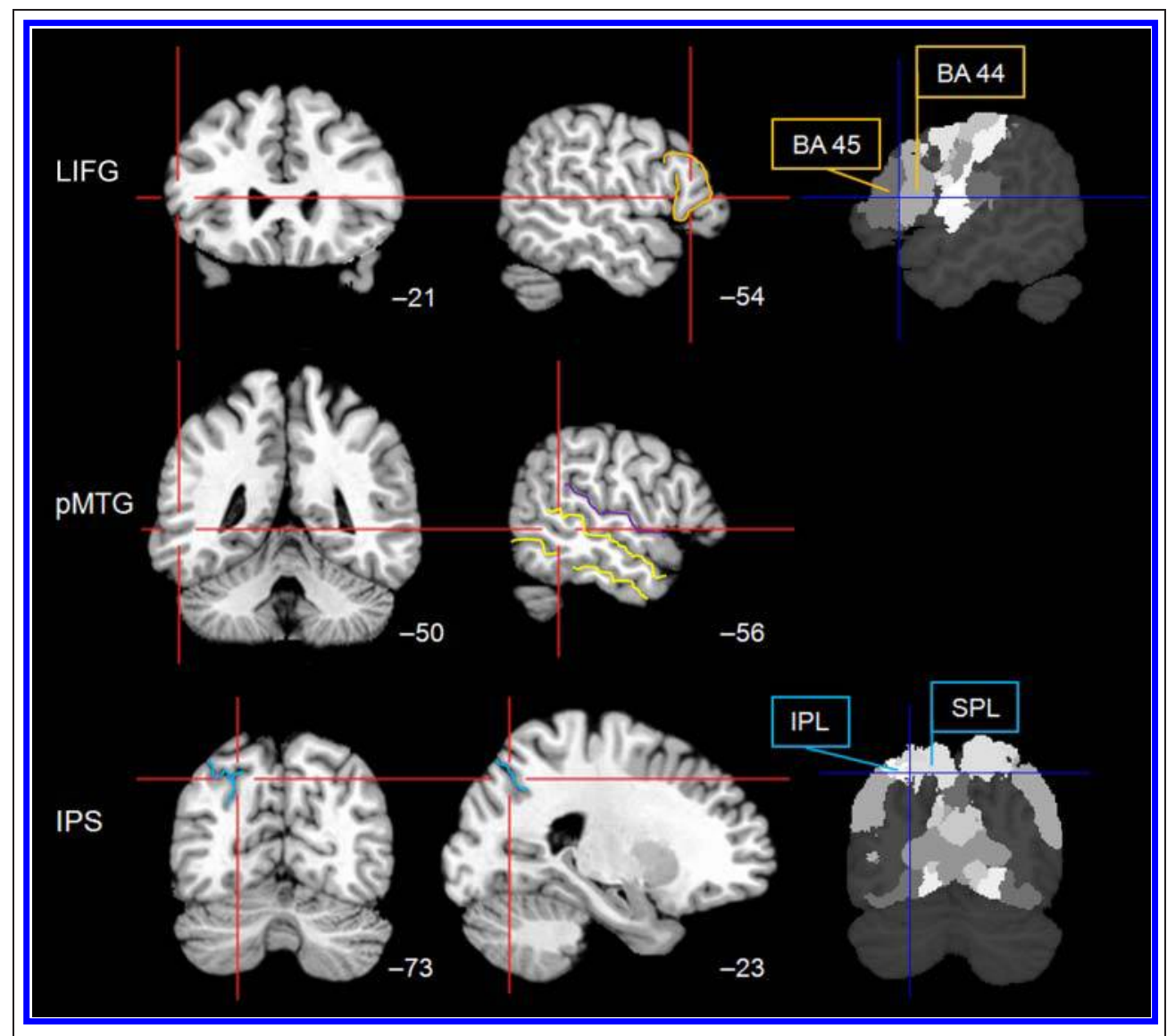


semantic manipulations including low vs. high relatedness, feature selection (as opposed to decisions based on low relatedness), and a task that manipulated target congruency during feature selection. Moreover, circumscribed lesions to this area resulted in poor performance during executively demanding semantic tasks in aphasic patients (Novick et al., 2009).

The location for left pMTG stimulation ( $-56-503)$ lay between the superior and inferior temporal sulcus and was slightly anterior to an imaginary line perpendicular to the most posterior horizontal segment of the Sylvian fissure (cf. Gennari et al., 2007; Figure 2). This site, in BA 21, was identified from the average MNI coordinates of two studies (Badre et al., 2005; Wagner et al., 2001), which both reported increased pMTG peak activity in response to verbal low vs. high relatedness judgments and when the number of response options was large as opposed to small. This area is frequently affected in patients with semantic control deficits following temporoparietal infarcts (Noonan et al., 2010).

MNI coordinates for left parietal lobe $(-23-7348)$ were mean values based on Thompson-Schill and colleagues' study (1997) and referred to an area close to the posterior bank of the IPS (BA 7; Figure 2). Enhanced parietal activation was observed during feature selection as opposed to high relatedness and when the response set was increased (see also Badre et al., 2005). Furthermore, damage to the inferior parietal lobule, reaching up to IPS, has been observed in some patients with SA (e.g., Noonan et al., 2010; Soni et al., 2009).

\section{Data Analysis}

The primary performance measure was RT because RT is sensitive to rTMS effects even in the absence of any decline in accuracy (cf. Lambon Ralph et al., 2009; Pobric et al., 2007; Devlin et al., 2003). RT data were screened for errors and outliers $( \pm 2 S D)$. We then employed two complementary analyses. In the first, we used ANOVAs to compare the impact of TMS across pairs of brain regions (i.e., LIFG vs. pMTG, LIFG vs. IPS, pMTG vs. IPS) for the semantic and Navon tasks separately. This is useful because a three-way interaction would confirm, in line with our predictions, that the impact of TMS was task- and site-specific, hence, that brain areas were functionally dissociable within the control network being tested. Second, we used $t$ tests to test specific hypotheses regarding the specificity of the TMS effects, establishing (i) which $\operatorname{task}(\mathrm{s})$ were significantly impaired and (ii) at what site(s). For this analysis, difference scores were calculated from post-TMS and baseline sessions for each subject in each condition at each site (i.e., the TMS effect). These planned $t$ test comparisons determined whether rTMS-induced effects were present (two-tailed one-sample $t$ test) and whether these effects were site-specific and task-specific (two-tailed paired $t$ tests). Error rates were analyzed using the same model.

\section{RESULTS}

\section{RT}

\section{ANOVAS}

We first considered data from the semantic conditions (high relatedness, low relatedness, feature selection) to examine which areas worked together to underpin semantic control. There was a significant three-way interaction between Site $(n=2)$, Task $(n=3)$, and TMS (baseline vs. post-TMS) when IPS was compared with LIFG and when IPS was compared with pMTG (see Table 1 and Figure 3). However, there were no interactions with Site when LIFG and pMTG were compared, suggesting that these regions are functionally dissociable from IPS but similar to each other in terms of their contribution to semantic control.

The second ANOVA compared the stimulation sites in a pairwise fashion for the two Navon tasks to establish whether any of the brain regions contributed to nonsemantic forms of control. Again, the results showed a three-way interaction between Site $(n=2)$, Task $(n=2)$, and TMS (baseline vs. post-TMS) for IPS versus LIFG and IPS versus pMTG, but not when LIFG and pMTG were compared (see Table 1 and Figure 3). These results suggest that IPS also dissociates from LIFG and PMTG in terms of nonsemantic functions.

\section{TMS Effects across Tasks}

We computed the size of the TMS effect for each task at each stimulation site (i.e., RT for post-TMS minus baseline performance; see Figure 4), allowing us to draw specific inferences about the functional role played by each area.

\section{LIFG}

rTMS over LIFG slowed performance during the low relatedness task (one-sample $t(15)=4.24, p=.001$ ) but not the high relatedness task (one-sample $t(15)<1$ ). Surprisingly, there was no significant TMS effect for the feature selection task (one-sample $t(15)<1$ ). Also, none of the Navon tasks was impaired after TMS (one-sample $t(15)<1$ ). The direct comparison between the effects for the high versus low relatedness condition was significant (paired $t(15)=3.35, p=$ $.004)$, supporting a role of LIFG in controlled semantic retrieval. Comparison between the two semantic tasks with high-control demands however revealed that these tasks were not dissociable (low relatedness vs. feature selection: paired $t(15)=1.13, p=.28)$.

\section{pMTG}

Stimulation of pMTG disrupted both semantic tasks with high-control demands (low relatedness: one-sample $t(15)=$ $2.51, p<.05$; feature selection: one-sample $t(15)=2.92$, $p=.01)$. There was no TMS effect for the semantic task with low-control demands (high relatedness: one-sample $t(15)<1)$. Again, both Navon tasks remained unaffected 
Table 1. $F$ and $p$ Values for the ANOVA for RT

\begin{tabular}{|c|c|c|c|c|c|c|c|}
\hline & Site & $T M S$ & Task & Site $\times$ TMS & Site $\times$ Task & TMS $\times$ Task & Site $\times$ TMS $\times$ Task \\
\hline \multicolumn{8}{|c|}{ Semantic Conditions Only (High Relatedness, Low Relatedness, Feature Selection) } \\
\hline$d f$ & 1,15 & 1,15 & 2,30 & 1,15 & 2,30 & 2,30 & 2,30 \\
\hline LIFG, IPS & $<1$ & 7.31 & 371.14 & $<1$ & $<1$ & 3.27 & 3.44 \\
\hline$p$ & .89 & .02 & $<.001$ & .97 & .64 & $.069^{\mathrm{a}}$ & .045 \\
\hline pMTG, IPS & $<1$ & 13.88 & 486.93 & $<1$ & $<1$ & 4.99 & 3.26 \\
\hline$p$ & .74 & .002 & $<.001$ & .42 & .63 & .014 & .05 \\
\hline LIFG, pMTG & $<1$ & 12.61 & 420.01 & $<1$ & 1.84 & 4.55 & $<1$ \\
\hline$p$ & .58 & .003 & $<.001$ & .48 & 1.76 & $.03^{\mathrm{a}}$ & .44 \\
\hline \multicolumn{8}{|c|}{ Nonsemantic Conditions Only (Global and Local Navon Task) } \\
\hline$d f$ & 1,15 & 1,15 & 1,15 & 1,15 & 1,15 & 1,15 & 1,15 \\
\hline LIFG, IPS & $<1$ & $<1$ & 99.32 & $<1$ & $<1$ & 4.34 & 10.01 \\
\hline$p$ & .74 & .46 & $<.001$ & .89 & .97 & .055 & .006 \\
\hline pMTG, IPS & $<1$ & 1.23 & 108.07 & $<1$ & 2.27 & $<1$ & 17.84 \\
\hline$p$ & .94 & .29 & $<.001$ & .67 & .15 & .53 & .001 \\
\hline LIFG, pMTG & $<1$ & $<1$ & 121.45 & $<1$ & 4.44 & 4.78 & 1.91 \\
\hline$p$ & .78 & .39 & $<.001$ & .69 & .052 & .045 & .19 \\
\hline
\end{tabular}

Pairs of brain regions that were compared are listed in the first column. The three-way interaction of the omnibus ANOVA, including all sites and conditions, was also significant $(F(8,120)=2.36 ; p=.05)$.

${ }^{\text {a }}$ Sphericity-corrected (Huynh-Feldt).

by TMS $(p>.09)$. The size of the TMS effects did not differ between the two high-control semantic conditions (paired $t(15)<1$ ), but both effects were larger compared with trials requiring minimal semantic control, indicating the importance of pMTG for various types of semantic control (low vs. high relatedness: paired $t(15)=2.41, p=.03$; feature selection vs. high relatedness: paired $t(15)=3.41$, $p=.004)$.

\section{IPS}

rTMS over IPS interfered with the feature selection task (one-sample $t(15)=3.35, p<.005$ ) and the global Navon task (one-sample $t(15)=3.86, p<.005$ ). The feature selection task was more impaired than the other two semantic conditions (feature selection vs. high relatedness: paired $t(15)=2.99, p=.01$; feature selection vs. low relatedness: paired $t(15)=2.24, p=.04)$, consistent with the specific function of IPS in top-down mediated selection. The global Navon task was also more affected than the local version (paired $t(15)=3.28, p=.005)$.

\section{TMS Effects across Sites}

The TMS effects for LIFG and pMTG in the low relatedness task were larger than the effect of TMS over IPS, which is in line with our prediction that IPS does not support con- trolled semantic retrieval (LIFG vs. IPS: paired $t(15)=2.55$, $p=.02$; pMTG vs. IPS: paired $t(15)=2.00, p=.06)$. There was no difference in the effect of TMS across LIFG and pMTG (paired $t(15)<1$ ), suggesting that both of these sites play a critical role in the controlled retrieval of semantic information (unlike IPS). In contrast, the TMS effect for the feature selection task did not differ across the three sites, implying that all targeted brain areas contribute to semantic selection (IPS vs. LIFG: paired $t(15)=1.41, p=$ .18; pMTG vs. LIFG: paired $t(15)=1.15, p=.27$; IPS vs. MTG: paired $t(15)<1$; see Figure 4). Finally, the TMS effect for the global Navon task was larger for IPS compared with LIFG or pMTG (IPS vs. LIFG: paired $t(15)=2.59, p=$ .02 ; IPS vs. pMTG: paired $t(15)=3.05, p=.008)$. This suggests, together with the findings from the ANOVA, that IPS contributes to nonsemantic decisions as well as to aspects of semantic control, unlike LIFG or pMTG.

In summary, the results of the ANOVAs showed that LIFG and pMTG performed similar functions, while IPS dissociated from both regions during semantic and nonsemantic control tasks (see Figures 3 and 4). Moreover, in line with our predictions, planned comparisons revealed that LIFG and pMTG exclusively mediated semantic control functions, while IPS contributed to control processes in both domains (semantic and nonsemantic; Figure 4). Differences also emerged regarding the type of semantic control that was supported by LIFG and pMTG as opposed to 


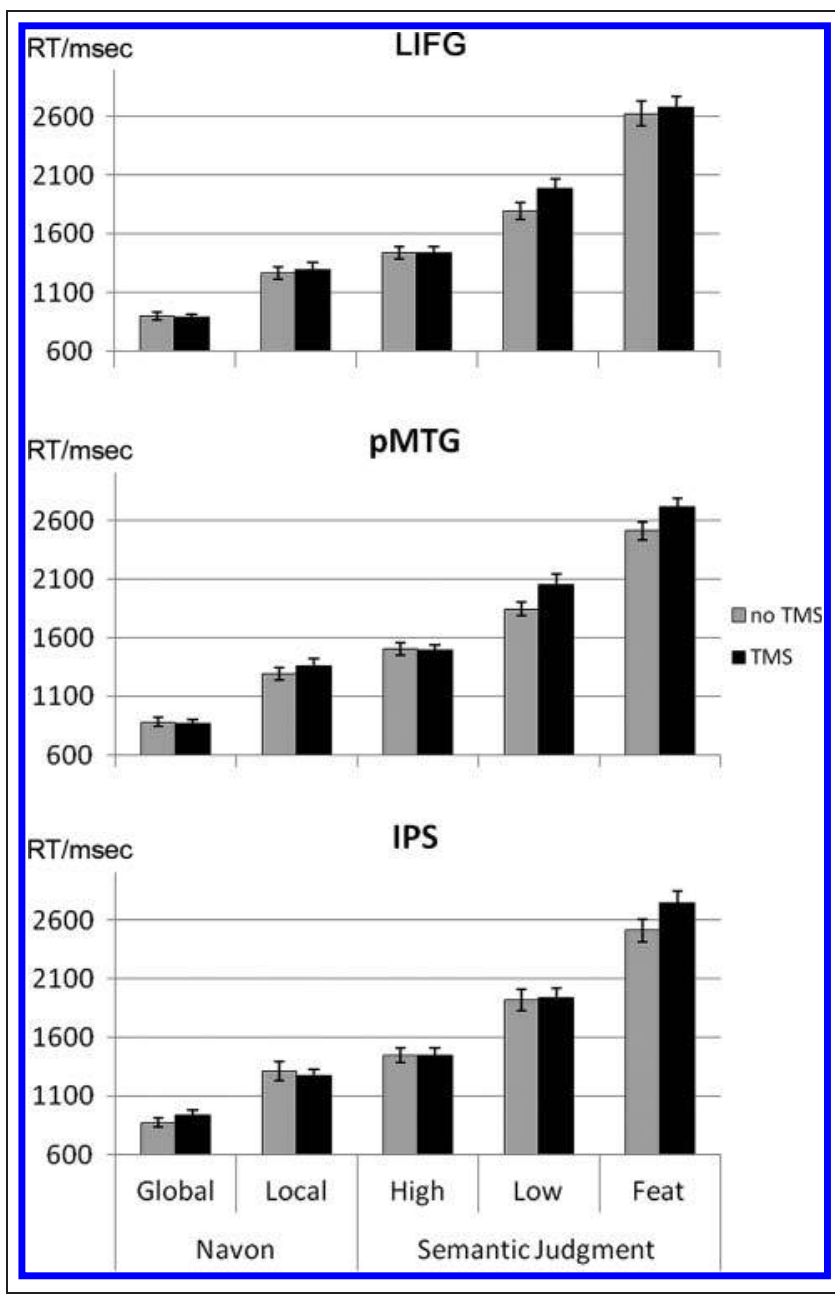

Figure 3. Reaction times. Performance at baseline (no TMS) and post-TMS following stimulation of LIFG, pMTG, and IPS. High = high relatedness; Low $=$ low relatedness; Feat $=$ feature selection. Error bars denote $S E M$

IPS: IPS was the only region that did not engage in controlled semantic retrieval but contributed to feature selection only.

Unexpectedly, TMS over IPS disrupted the easier global Navon task, although fMRI studies have shown increased brain activity in the left IPS when participants attended to local and less-salient dimensions of Navon letters (corresponding to the Local Navon task; Mevorach et al., 2008). One possibility is that the intended automatic mapping of cue and target letter during the global Navon task was hindered by our use of different fonts, which meant that sometimes the shape of the cue deviated significantly from the global shape of the target compound and could be ambiguous (e.g., a lower-case "e" in font "Curlz" could be mistaken for a "c"). Therefore, the global Navon task might have required a stronger level of cognitive control than expected. Simultaneously, these manipulations had the opposite effect on the control requirements of the local Navon task. Here, a globally related letter was used as one of the distractor items, which was meant to act as a highly competitive but irrelevant response (e.g., when "c" was the cue and a large " $C$ " was the distractor). If the automatic association between globally identical cue and target letters was reduced by the use of different fonts, strong inhibition processes may have no longer been required, which might explain the lack of a TMS effect for the local Navon task.

\section{Error Rates}

\section{ANOVAs}

Table 2 lists the error rates that were entered into the ANOVAs. These analyses revealed that no effects were driven by Site in any of the six ANOVAs, which compared pairs of brain regions separately for semantic and Navon tasks (as in the RT analysis; Table 3). Instead, TMS effects were dependent on the tasks in the semantic conditions (i.e., the TMS $\times$ Task interaction was significant in all three ANOVAs comparing pairs of brain regions), while TMS had no influence on performance of the Navon tasks (i.e., no main effects or interactions with TMS were observed; $F<1)$.

\section{Planned Comparisons}

Planned comparisons on the difference scores between post-TMS and baseline performance were used to test specific predictions about the impact of TMS on task performance, separately for each target area. Two effects

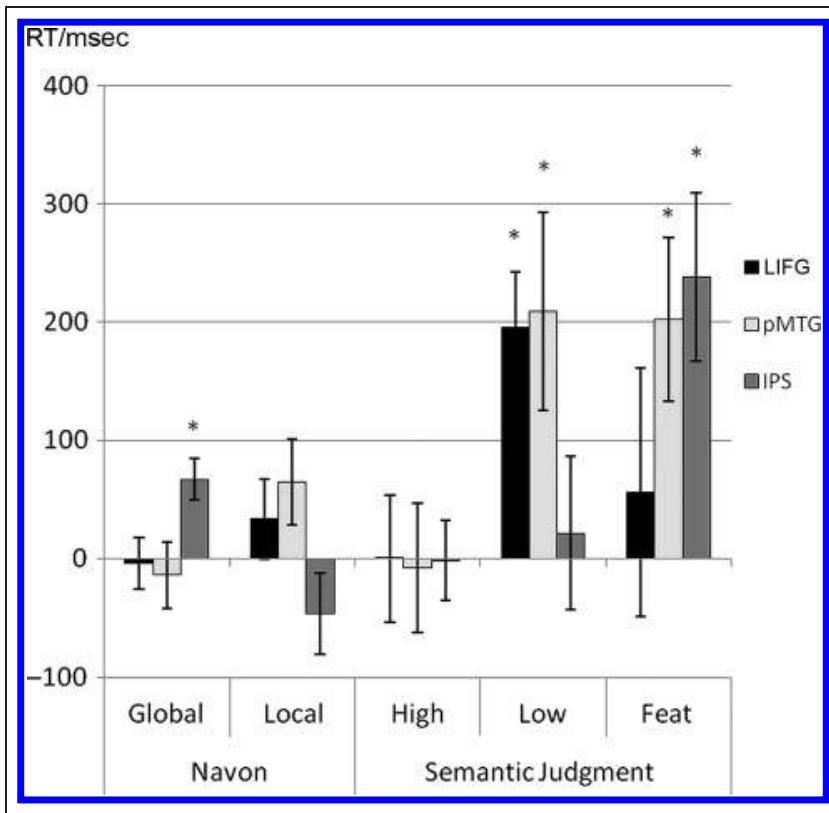

Figure 4. TMS effect. Difference scores between post-TMS and baseline performance (TMS-no TMS) for reaction time. Positive values indicate a decline in performance after brain stimulation, whereas negative values indicate improvement. High $=$ high relatedness; Low $=$ low relatedness; Feat $=$ feature selection . $* p<.05$. Error bars denote SEM. 
Table 2. Error Rates

\begin{tabular}{|c|c|c|c|c|c|}
\hline & High Relatedness & Low Relatedness & Feature Selection & Global Navon & Local Navon \\
\hline \multicolumn{6}{|l|}{$L I F G$} \\
\hline No TMS & $2.00(2.42)$ & $5.75(4.91)$ & $17.31(10.01)$ & $2.63(3.07)$ & $4.75(12.31)$ \\
\hline TMS & $3.13(2.99)$ & $11.25(6.44)$ & $12.25(8.51)$ & $1.81(2.97)$ & $4.38(3.50)$ \\
\hline Difference & $1.13(3.74)$ & $5.50(8.63)$ & $-5.06(11.32)$ & $-0.82(4.82)$ & $-0.37(12.96)$ \\
\hline \multicolumn{6}{|l|}{ pMTG } \\
\hline No TMS & $2.94(2.59)$ & $7.44(6.31)$ & $15.06(7.86)$ & $1.19(2.04)$ & $3.44(5.25)$ \\
\hline TMS & $3.25(3.66)$ & $11.25(5.69)$ & $13.13(8.37)$ & $1.50(1.55)$ & $2.81(2.48)$ \\
\hline Difference & $0.31(3.94)$ & $3.81(7.47)$ & $-1.93(8.23)$ & $0.31(2.82)$ & $-0.63(4.47)$ \\
\hline \multicolumn{6}{|l|}{ IPS } \\
\hline No TMS & $2.81(3.97)$ & $9.06(8.81)$ & $20.94(10.85)$ & $1.81(2.46)$ & $7.38(13.16)$ \\
\hline TMS & $5.38(4.21)$ & $9.06(4.93)$ & $15.81(15.01)$ & $1.94(2.62)$ & $4.00(5.27)$ \\
\hline Difference & $2.57(6.03)$ & $0.00(6.68)$ & $-5.13(10.76)$ & $0.13(3.52)$ & $-3.38(13.68)$ \\
\hline
\end{tabular}

Percentage of errors committed during performance at baseline (no TMS) and after TMS (TMS) to LIFG, pMTG, and IPS for each task separately. Difference scores (TMS-no TMS) reflect the TMS effect, with positive values indicating a decline in performance after brain stimulation whereas negative values signal improvement. Standard deviation is given in parentheses.

Table 3. $F$ and $p$ Values for the ANOVA for Error Rate

\begin{tabular}{|c|c|c|c|c|c|c|c|}
\hline & Site & $T M S$ & Task & Site $\times$ TMS & Site $\times$ Task & TMS $\times$ Task & Site $\times$ TMS $\times$ Task \\
\hline \multicolumn{8}{|c|}{ Semantic Conditions Only (High Relatedness, Low Relatedness, Feature Selection) } \\
\hline$d f$ & 1,15 & 1,15 & 2,30 & 1,15 & 2,30 & 2,30 & 2,30 \\
\hline LIFG, IPS & 2.89 & $<1$ & 35.49 & 1.05 & $<1$ & 6.29 & 2.07 \\
\hline$p$ & .11 & .86 & $<.001^{\mathrm{a}}$ & .32 & .44 & $.014^{\mathrm{a}}$ & $.16^{\mathrm{a}}$ \\
\hline pMTG, IPS & 3.12 & $<1$ & 27.75 & $<1$ & 2.65 & 4.42 & 44.74 \\
\hline$p$ & .10 & .94 & $<.001$ & .34 & .09 & $.036^{\mathrm{a}}$ & .10 \\
\hline LIFG, pMTG & $<1$ & $<1$ & 41.34 & $<1$ & $<1$ & 7.58 & 1.01 \\
\hline$p$ & .70 & .44 & $<.001^{\mathrm{a}}$ & .89 & .68 & $.008^{\mathrm{a}}$ & .38 \\
\hline \multicolumn{8}{|c|}{ Nonsemantic Conditions Only (Global and Local Navon Task) } \\
\hline$d f$ & 1,15 & 1,15 & 1,15 & 1,15 & 1,15 & 1,15 & 1,15 \\
\hline LIFG, IPS & $<1$ & $<1$ & 6.66 & $<1$ & $<1$ & $<1$ & $<1$ \\
\hline$p$ & .76 & .36 & .02 & .67 & .56 & .51 & .51 \\
\hline pMTG, IPS & 2.44 & 1.82 & 6.31 & $<1$ & 1.27 & 1.30 & $<1$ \\
\hline$p$ & .14 & .20 & .02 & .46 & .28 & .27 & .57 \\
\hline LIFG, pMTG & 1.49 & $<1$ & 4.65 & $<1$ & $<1$ & $<1$ & $<1$ \\
\hline$p$ & .24 & .72 & .048 & .82 & .77 & .89 & .68 \\
\hline
\end{tabular}

Pairs of brain regions that were compared are listed in the first column.

${ }^{a}$ Sphericity-corrected (Huynh-Feldt). 
emerged, supporting the RT results: Error rates increased for the low relatedness condition after TMS was applied to LIFG (one-sample $t(15)=2.55, p<.05$ ), and this effect also approached significance for pMTG (one-sample $t(15)=2.04, p=.06)$. When comparing the TMS effect across sites, the only difference was between LIFG and IPS (paired $t(15)=2.09, p=.05$ ). This reflected the fact that TMS to LIFG, but not IPS, disrupted controlled semantic retrieval, mirroring the $\mathrm{RT}$ data.

\section{DISCUSSION}

This study used rTMS to examine the roles of LIFG, pMTG, and IPS in two different forms of semantic control, that is, stimulus driven, controlled retrieval, and top-down selection of semantic knowledge. Neuroimaging studies and semantically impaired patients with SA both indicate that LIFG plays a crucial role in semantic control, but there is little consensus on (1) the possible roles of left pMTG and parietal cortex, (2) the extent to which different aspects of semantic control are mediated by different cortical regions, and (3) the relationship between semantic control and cognitive control more widely. TMS over all three regions led to specific disruption of at least one aspect of semantic control, indicating that controlled retrieval and semantic selection are not exclusively mediated by LIFG. Furthermore, depending on the type of control function, different components of this fronto-temporo-parietal network played a crucial role. Although LIFG and pMTG participated in controlled meaning retrieval, pMTG worked in concert with IPS to compensate for increased semantic selection demands (our data are equivocal on the potential role of LIFG in semantic selection; see Figure 4 and below). The roles of LIFG and pMTG were largely indistinguishable and restricted to executively demanding semantic judgments, while TMS over IPS also disrupted the nonsemantic Navon task.

\section{The Role of LIFG in Semantic Control}

The finding that LIFG supports semantic judgments when executive demands are high is well established in both the neuropsychological and neuroimaging literature. Patients with damage to LIFG after SA are impaired across a variety of semantic control manipulations, including some of the tasks used in the current study (i.e., relatedness judgments with strong and weakly related targets; Novick et al., 2009; Jefferies \& Lambon Ralph, 2006; Thompson-Schill et al., 1998). fMRI work is largely consistent with these findings, although closer inspection of the activation patterns in these studies suggests a degree of functional specialization within LIFG, with semantic selection and controlled retrieval relying on different substructures (Badre \& Wagner, 2007; Snyder, Feigenson, \& Thompson-Schill, 2007; Zempleni, Renken, Hoeks, Hoogduin, \& Stowe, 2007; Badre et al., 2005; Noppeney et al., 2004; Thompson-Schill et al., 1997). According to this view, dorsal aspects of BA 45, which cor- respond to the target area in this study, have been linked to tasks that require the selection of a subset of information from among competitors in the semantic store (e.g., when attention was drawn to obscure features or when more than one word meaning competed for selection during ambiguity resolution). In contrast, ventral portions of LIFG (BA 45/BA 47) were engaged in semantic control tasks with low selection requirements (e.g., during the controlled retrieval of semantic knowledge). Our TMS data do not support this distinction, because stimulation of dorsal BA 45 disrupted both high-control tasks.

At the moment, it is unclear which aspects of the control process drive the dissociation in prefrontal cortex: We have already indicated that the feature selection task differs from the low relatedness tasks in terms of its requirement for top-down as opposed to bottom-up control, which is a highly relevant distinction for predicting activation in parietal cortex (Ciaramelli et al., 2008). Moreover, the functional organization of prefrontal cortex has been described along different dimensions, for example, processes were grouped according to the complexity of the decisions and representations involved rather than whether selection was a critical component or not (Badre, 2008; Badre \& D’Esposito, 2007; Koechlin, Ody, \& Kouneiher, 2003). Finally, there exists large variability in the anatomy of prefrontal cortex, with little correspondence across individuals to macroanatomical landmarks used to define the boarders of BA 44 and BA 45, hence hindering the attribution of cognitive functions to specific microanatomical structures (Amunts et al., 1999).

Our data are more consistent with the general view that BA 45 forms part of the ventral stream for language processing ("semantic pathway"): that is, along with other regions, it mediates processes that support the search for and selection of target words and concepts stored elsewhere in the brain, because this requirement is inherent to both of our semantic control tasks (Heim, Eickhoff, \& Amunts, 2009; Heim, Eickhoff, Ischebeck, et al., 2009; Saur et al., 2008; Heim et al., 2005; Amunts et al., 2004; Hickok \& Poeppel, 2004; Fiebach, Friederici, Muller, \& von Cramon, 2002). Research based on anatomically precise cytoarchitectonic maps has shown that activation increased in BA 45 when the semantic search was effortful and exhaustive, following manipulations of the lexicosemantic properties of the word (e.g., stronger for low as opposed to high-frequency words; pseudowords > words; Heim et al., 2005; Fiebach et al., 2002). Activation in BA 45 also fluctuated with the degree of semantic processing involved (e.g., semantic vs. phonological fluency or decisions; Heim, Eickhoff, Ischebeck, et al., 2009; Amunts et al., 2004). In contrast, changes in phonological stimulus attributes or task demands altered activity in BA 44, which is a brain region implicated in the dorsal language pathway ("phonological route"; Heim, Eickhoff, \& Amunts, 2009; Saur et al., 2008; Hickok \& Poeppel, 2004). In line with these data, a previous study has shown that TMS over BA 44 impaired phonological judgments and spared 
semantic decisions, while the reverse behavioral pattern was observed after stimulation of BA 45/BA 47 (Gough, Nobre, \& Devlin, 2005). Our study extends these findings, showing that activity in BA 45 is causally linked to the level of semantic control needed to retrieve the target concept.

\section{The Role of Left pMTG in Semantic Control}

The performance deficits observed after left pMTG stimulation were indistinguishable from the effects of TMS over LIFG, suggesting that pMTG and LIFG work together as part of a distributed executive semantic network supporting semantic selection and controlled retrieval. These results are consistent with neuropsychological studies of patients with SA, who show similar performance deficits on tasks that tap semantic control following either damage to temporo-parietal areas, including pMTG, or in combination with LIFG (Corbett, Jefferies, \& Lambon Ralph, 2011; Noonan et al., 2010; Jefferies \& Lambon Ralph, 2006). Using voxel-based lesion symptom mapping, Schwartz and colleagues (2009) demonstrated that naming deficits in aphasia patients could not be attributed to damage in LIFG or posterior temporal cortex once executive semantic control processes were controlled for. In contrast, the contribution of other "semantic" regions in more anterior temporal lobe was not linked to semantic control. Both of these lines of research suggest that left pMTG and LIFG perform similar semantic control functions; however, up until now, there was little direct evidence that selective disruption of pMTG and LIFG can produce equivalent deficits in semantic control.

The TMS results further resolve some of the ambiguity from the neuroimaging literature regarding pMTG functioning, which has linked activation in this area to confounding increases in representational processes during tasks with high semantic control requirements (Bedny, McGill, \& Thompson-Schill, 2008; Badre et al., 2005; Gold \& Buckner, 2002; Wagner et al., 2001). For example, in the feature selection task, strongly associated but task-irrelevant concepts are activated alongside the target item (via automatic spreading of activation in the semantic store). Because in our study, deficits in semantic control were observed as a direct consequence of temporary disruption to pMTG, it is no longer plausible to suggest that brain activation in pMTG during fMRI is a by-product rather than a causal consequence of manipulations in semantic control processes. Further support is provided by severely aphasic patients with lesions to pMTG who are asked to perform sentence-picture matching tasks with different levels of difficulty. Comprehension is best when the meaning of the sentence can be derived from the high-frequency nouns alone, hence demonstrating spared semantic knowledge, compared with sentences where understanding the more complex verb/verb argument structure is crucial (Dronkers, Wilkins, Van Valin, Redfern, \& Jaeger, 2004).

An alternative proposal is that pMTG acts as a semantic store that encodes specific semantic attributes, associated only with a subset of the stimuli tested in our experiment (i.e., motion attributes; Wallentin et al., 2011; Dick, Goldin-Meadow, Hasson, Skipper, \& Small, 2009; Martin, 2007; Damasio, Grabowski, Tranel, Hichwa, \& Damasio, 1996). It seems unlikely that this could explain why pMTG stimulation specifically disrupted the control-demanding semantic tasks and not the low-control condition, because all three semantic tasks included stimuli from a wide range of categories. Moreover, pMTG showed activation during fMRI for the same stimuli, suggesting that even if pMTG activation is modulated by semantic category/feature, this site does not have a single role tightly restricted to a specific category. Further research is clearly needed to establish whether the same pMTG region responds to semantic control demands and feature manipulations, such as action judgments, and if so, why.

Apart from pMTG, other parts of temporal cortex have been linked to storing semantic representation, including more anterior and inferior temporal cortex (for reviews, see Binder et al., 2009; Patterson, Nestor, \& Rogers, 2007). Bilateral atrophy focused on anterior inferior temporal cortex results in a gradual degradation of semantic knowledge, as seen in patients with semantic dementia (Binney, Embleton, Jefferies, Parker, \& Lambon Ralph, 2010; Hodges \& Patterson, 2007; Jefferies \& Lambon Ralph, 2006; Mummery et al., 2000; Hodges, Patterson, Oxbury, \& Funnell, 1992). The impairment in these patients is highly consistent across tasks with varying control demands and depends on factors that describe the complexity of the semantic representation being retrieved, such as familiarity and typicality. In contrast, in individuals with semantic control deficits, lesions affect left prefrontal, posterior temporal, and parietal structures, while the anterior temporal lobe is spared, and conceptual knowledge remains accessible once semantic control requirements are reduced (e.g., Jefferies et al., 2008; Jefferies \& Lambon Ralph, 2006). This double dissociation between executive and representational aspects of semantic cognition in the left temporal cortex was confirmed in a recent fMRI investigation (Whitney, Jefferies, \& Kircher, 2011). Semantic selection requirements were linked to LIFG, pMTG, and parietal cortex, while manipulations of the number of meanings likely to be activated in a trial loaded onto more anterior and inferior parts of left temporal lobe (i.e., BA 20). Together, these observations imply that semantic representation and control processes rely on different regions within left temporal cortex.

\section{The Role of Left IPS in Semantic and Nonsemantic Forms of Control}

The function of left IPS in the control network was distinct from that of LIFG and pMTG in two ways. First, IPS was the only brain region that responded solely to manipulations in semantic selection requirements as opposed to controlled meaning retrieval. This response pattern was expected based on studies of attention that implicated IPS in tasks that required top-down control, elicited by explicit 
cues meant to bias behavior even before stimulus presentation (here: e.g., attend to color), as opposed to control functions that are driven by the stimulus and are not cued (Ciaramelli et al., 2008). Second, participation of IPS was independent of whether semantic or nonsemantic stimuli were used. rTMS affected response times in both the feature selection and the global Navon task, indicating that IPS plays a wider role in cognition than either LIFG or pMTG.

Neuroimaging data reveals that IPS, unlike LIFG and pMTG, is a brain region that forms part of a fronto-parietal MD network, supporting tasks that require high executive control, independently of stimulus modality (although semantic control, as it is examined here, has not been explicitly tested; Duncan, 2010; Duncan \& Owen, 2000; Owen et al., 2000). Furthermore, semantically impaired patients with lesions to temporo-parietal cortex, including IPS, suffer from executive deficits that go far beyond the semantic domain (Corbett, Jefferies, Ehsan, et al., 2009; Jefferies \& Lambon Ralph, 2006). The strongest support, however, for a distinction between MD regions (in and around IPS) and semantic-specific control areas (situated in inferior frontal and posterior temporal cortex) comes from fMRI research that has directly compared semantic and nonsemantic executive functions. Consistently, these results point toward an engagement of the left IPS in any form of control (Binney et al., 2010; Nagel, Schumacher, Goebel, \& D’Esposito, 2008; Cristescu et al., 2006). In contrast, activation in LIFG and pMTG is limited to tasks with high semantic control demands. Using TMS, we were able to verify these observations and clearly establish a dissociation between components in the semantic control network: Although all three areas (LIFG, pMTG, IPS) were important for semantic aspects of control, only IPS contributed to executive functions beyond the semantic domain.

\section{Acknowledgments}

This work was supported by a Wellcome project grant (078734/ Z05/Z to E. J. and M. A. L. R.). We thank David Badre, Peter Hills, and Michael Lewis for supplying their stimulus materials.

Reprint requests should be sent to Carin Whitney or Elizabeth Jefferies, Department of Psychology, University of York, YO10 5DD, York, United Kingdom, or via e-mail: c.whitney@psych.york.ac.uk; ej514@york.ac.uk.

\section{REFERENCES}

Amunts, K., Schleicher, A., Burgel, U., Mohlberg, H., Uylings, H. B., \& Zilles, K. (1999). Broca's region revisited: Cytoarchitecture and intersubject variability. Lournal of Comparative Neurology, 412, 319-341.

Amunts, K., Weiss, P. H., Mohlberg, H., Pieperhoff, P., Eickhoff, S., Gurd, J. M., et al. (2004). Analysis of neural mechanisms underlying verbal fluency in cytoarchitectonically defined stereotaxic space-The roles of Brodmann areas 44 and 45 . Neuroimage, 22, 42-56.

Badre, D. (2008). Cognitive control, hierarchy, and the rostro-caudal organization of the frontal lobes. Trends in Cognitive Sciences, 12, 193-200.
Badre, D., \& D'Esposito, M. (2007). Functional magnetic resonance imaging evidence for a hierarchical organization of the prefrontal cortex. Journal of Cognitive Neuroscience, 19, 2082-2099.

Badre, D., Poldrack, R. A., Pare-Blagoev, E. J., Insler, R. Z., \& Wagner, A. D. (2005). Dissociable controlled retrieval and generalized selection mechanisms in ventrolateral prefrontal cortex. Neuron, 47, 907-918.

Badre, D., \& Wagner, A. D. (2007). Left ventrolateral prefrontal cortex and the cognitive control of memory. Neuropsychologia, 45, 2883-2901.

Bedny, M., McGill, M., \& Thompson-Schill, S. L. (2008). Semantic adaptation and competition during word comprehension. Cerebral Cortex, 18, 2574-2585.

Billington, J., Baron-Cohen, S., \& Bor, D. (2008). Systemizing influences attentional processes during the Navon task: An fMRI study. Neuropsvchologia. 46, 511-520.

Binder, J. R., Desai, R. H., Graves, W. W., \& Conant, L. L. (2009). Where is the semantic system? A critical review and meta-analysis of 120 functional neuroimaging studies. Cerebral Cortex, 19, 2767-2796.

Binney, R. J., Embleton, K. V., Jefferies, E., Parker, G. J., \& Lambon Ralph, M. A. (2010). The ventral and inferolateral aspects of the anterior temporal lobe are crucial in semantic memory: Evidence from a novel direct comparison of distortion-corrected fMRI, rTMS, and semantic dementia. Cerebral Cortex, 20, 2728-2738.

Ciaramelli, E., Grady, C. L., \& Moscovitch, M. (2008). Top-down and bottom-up attention to memory: A hypothesis (AtoM) on the role of the posterior parietal cortex in memory retrieval. Neuropsychologia, 46, 1828-1851.

Collette, F., Hogge, M., Salmon, E., \& Van der Linden, M. (2006). Exploration of the neural substrates of executive functioning by functional neuroimaging. Neuroscience, 139, 209-221.

Collins, A. M., \& Loftus, E. F. (1975). Spreading activation theory of semantic processing. Psvchological Review, 82, 407-428.

Corbett, F., Jefferies, E., Ehsan, S., \& Lambon Ralph, M. A. (2009). Different impairments of semantic cognition in semantic dementia and semantic aphasia: Evidence from the non-verbal domain. Brain, 132, 2593-2608.

Corbett, F., Jefferies, E., \& Lambon Ralph, M. A. (2009). Exploring multimodal semantic control impairments in semantic aphasia: Evidence from naturalistic object use. Neuropsychologia, 47, 2721-2731.

Corbett, F., Jefferies, E., \& Lambon Ralph, M. A. (2011). Deregulated semantic cognition follows prefrontal and temporo-parietal damage: Evidence from the impact of task constraint on nonverbal object use. Journal of Cognitive Neuroscience, 23, 1125-1135.

Cristescu, T. C., Devlin, J. T., \& Nobre, A. C. (2006). Orienting attention to semantic categories. Neuroimage 33, 1178-1187.

Damasio, H., Grabowski, T. J., Tranel, D., Hichwa, R. D., \& Damasio, A. R. (1996). A neural basis for lexical retrieval. Nature, 380, 499-505.

Devlin, J. T., Matthews, P. M., \& Rushworth, M. F. (2003). Semantic processing in the left inferior prefrontal cortex: A combined functional magnetic resonance imaging and transcranial magnetic stimulation study. Journal of Cognitive Neuroscience, 15, 71-84.

Devlin, J. T., \& Watkins, K. E. (2007). Stimulating language: Insights from TMS. Brain, 130, 610-622.

Dick, A. S., Goldin-Meadow, S., Hasson, U., Skipper, J. I., \& Small, S. L. (2009). Co-speech gestures influence neural activity in brain regions associated with processing semantic information. Human Brain Mapping, 30, 3509-3526. 
Dronkers, N. F., Wilkins, D. P., Van Valin, R. D., Jr., Redfern, B. B., \& Jaeger, J. J. (2004). Lesion analysis of the brain areas involved in language comprehension. Cognition. 92, 145-177.

Duncan, J. (2006). EPS Mid-Career Award 2004: Brain mechanisms of attention. Ouarterly Iournal of Experimental Psychologv: (Colchester), 59, 2-27.

Duncan, J. (2010). The multiple-demand (MD) system of the primate brain: Mental programs for intelligent behavior. Trends in Cognitive Sciences, 14, 172-179.

Duncan, J., \& Owen, A. M. (2000). Common regions of the human frontal lobe recruited by diverse cognitive demands. Trends in Neurosciences, 23, 475-483.

Fiebach, C. J., Friederici, A. D., Muller, K., \& von Cramon, D. Y. (2002). fMRI evidence for dual routes to the mental lexicon in visual word recognition. Journal of Cognitive Neuroscience, 14, 11-23.

Gennari, S. P., MacDonald, M. C., Postle, B. R., \& Seidenberg, M. S. (2007). Context-dependent interpretation of words: Evidence for interactive neural processes. Neuroimage, $35,1278-1286$

Gold, B. T., \& Buckner, R. L. (2002). Common prefrontal regions coactivate with dissociable posterior regions during controlled semantic and phonological tasks. Neuron, 35, 803-812.

Gough, P. M., Nobre, A. C., \& Devlin, J. T. (2005). Dissociating linguistic processes in the left inferior frontal cortex with transcranial magnetic stimulation. Dournal of Neuroscience 25, 8010-8016.

Hedden, T., \& Gabrieli, J. D. (2010). Shared and selective neural correlates of inhibition, facilitation, and shifting processes during executive control. Neuroimage, 51, 421-431.

Heim, S., Alter, K., Ischebeck, A. K., Amunts, K., Eickhoff, S. B., Mohlberg, H., et al. (2005). The role of the left Brodmann's areas 44 and 45 in reading words and pseudowords. Cognitive Brain Research, 25, 982-993.

Heim, S., Eickhoff, S. B., \& Amunts, K. (2009). Different roles of cytoarchitectonic BA 44 and BA 45 in phonological and semantic verbal fluency as revealed by dynamic causal modelling. Neuroimage, 48, 616-624.

Heim, S., Eickhoff, S. B., Ischebeck, A. K., Friederici, A. D., Stephan, K. E., \& Amunts, K. (2009). Effective connectivity of the left BA 44, BA 45, and inferior temporal gyrus during lexical and phonological decisions identified with DCM. Human Brain Mapping, 30, 392-402.

Hickok, G., \& Poeppel, D. (2004). Dorsal and ventral streams: A framework for understanding aspects of the functional anatomy of language. Cognition, 92, 67-99.

Hills, P. J., \& Lewis, M. B. (2007). Temporal limitation of Navon effect on face recognition. Perceptual and Motor Skills, 104, 501-509.

Hodges, J. R., \& Patterson, K. (2007). Semantic dementia: A unique clinicopathological syndrome. Lancet Neurology 6, 1004-1014

Hodges, J. R., Patterson, K., Oxbury, S., \& Funnell, E. (1992). Semantic dementia. Progressive fluent aphasia with temporal lobe atrophy. Brain, 115, 1783-1806.

Indefrey, P., \& Levelt, W. J. (2004). The spatial and temporal signatures of word production components. Cognition. 92, 101-144.

Jefferies, E., Baker, S. S., Doran, M., \& Lambon Ralph, M. A. (2007). Refractory effects in stroke aphasia: A consequence of poor semantic control. Neuropsychologia, 45, 1065-1079.

Jefferies, E., \& Lambon Ralph, M. A. (2006). Semantic impairment in stroke aphasia versus semantic dementia: A case-series comparison. Brain, 129, 2132-2147.

Jefferies, E., Patterson, K., \& Lambon Ralph, M. A. (2008). Deficits of knowledge versus executive control in semantic cognition: Insights from cued naming. Neuropsychologia 46, 649-658.

Keller, S. S., Crow, T., Foundas, A., Amunts, K., \& Roberts, N. (2009). Broca's area: Nomenclature, anatomy, typology and asymmetry. Brain and Language, 109, 29-48.

Koechlin, E., Ody, C., \& Kouneiher, F. (2003). The architecture of cognitive control in the human prefrontal cortex. Science, 302, 1181-1185.

Kucera, H., \& Francis, W. N. (1967). Computational analysis of present-day English. Providence, RI: Brown University Press.

Lambon Ralph, M. A., Pobric, G., \& Jefferies, E. (2009). Conceptual knowledge is underpinned by the temporal pole bilaterally: Convergent evidence from rTMS. Cerebral Cortex, 19, 832-838.

Martin, A. (2007). The representation of object concepts in the brain. Annual Review of Psychology, 58, 25-45.

Masson, M. (1991). A distributed memory model of context effects in word identification. In D. Besner \& G. W. Humphreys (Eds.), Basic processes in reading: Visual word recognition (pp. 233-263). Hilldale, NJ: Lawrence Erlbaum Associates.

Mevorach, C., Humphreys, G. W., \& Shalev, L. (2005). Attending to local form while ignoring global aspects depends on handedness: Evidence from TMS. Nature Neuroscience, 8, 276-277.

Mevorach, C., Shalev, L., Allen, H. A., \& Humphreys, G. W. (2008). The left intraparietal sulcus modulates the selection of low salient stimuli. Journal of Cognitive Neuroscience, 21, 303-315.

Moss, H., \& Older, L. (1996). Birbeck word association norms. Hove, UK: Psychology Press.

Mummery, C. J., Patterson, K., Price, C. J., Ashburner, J., Frackowiak, R. S., \& Hodges, J. R. (2000). A voxel-based morphometry study of semantic dementia: Relationship between temporal lobe atrophy and semantic memory. Annals of Neurologv, 47, 36-45.

Nagel, I. E., Schumacher, E. H., Goebel, R., \& D’Esposito, M. (2008). Functional MRI investigation of verbal selection mechanisms in lateral prefrontal cortex. Neuroimage, 43, 801-807.

Navon, D. (1977). Forest before trees: The precedence of global features in visual perception. Cognitive Psychology, 9, 353-383.

Neely, J. (1990). Semantic priming in visual word recognition: A selective review of current theories and findings. In D. Besner \& G. W. Humphreys (Eds.), Basic processes in reading: Visual word recognition (pp. 264-336). Hillsdale, NJ: Lawrence Erlbaum Associates.

Niyazov, D. M., Butler, A. J., Kadah, Y. M., Epstein, C. M., \& $\mathrm{Hu}$, X. P. (2005). Functional magnetic resonance imaging and transcranial magnetic stimulation: Effects of motor imagery, movement and coil orientation. Clinical Neuropbysiology, 116, 1601-1610.

Noonan, K. A., Jefferies, E., Corbett, F., \& Lambon Ralph, M. A. (2010). Elucidating the nature of deregulated semantic cognition in semantic aphasia: Evidence for the roles of prefrontal and temporo-parietal cortices. Journal of Cognitive Neuroscience, 22, 1597-1613.

Noppeney, U., Phillips, J., \& Price, C. (2004). The neural areas that control the retrieval and selection of semantics. Neuropsychologia, 42, 1269-1280.

Novick, J. M., Kan, I. P., Trueswell, J. C., \& Thompson-Schill, S. L. (2009). A case for conflict across multiple domains: Memory and language impairments following damage to ventrolateral prefrontal cortex. Cognitive Neuropsychology. 26, 527-567.

Owen, A. M., Schneider, W. X., \& Duncan, J. (2000). Executive control and the frontal lobe: Current issues. Experimental Brain Research, 133, 1-2. 
Pascual-Leone, A., Tormos, J. M., Keenan, J., Tarazona, F., Canete, C., \& Catala, M. D. (1998). Study and modulation of human cortical excitability with transcranial magnetic stimulation. Iournal of Clinical Neuropbysiology, 15, 333-343.

Pascual-Leone, A., Walsh, V., \& Rothwell, J. (2000). Transcranial magnetic stimulation in cognitive neuroscience-Virtual lesion, chronometry, and functional connectivity. Current Opinion in Neurobiology, 10, 232-237.

Patterson, K., Nestor, P. J., \& Rogers, T. T. (2007). Where do you know what you know? The representation of semantic knowledge in the human brain. Nature Reviews Neuroscience, 8, 976-987.

Pobric, G., Jefferies, E., \& Lambon Ralph, M. A. (2007). Anterior temporal lobes mediate semantic representation: Mimicking semantic dementia by using rTMS in normal participants. Proceedings of the National Academv of Sciences. U.S.A., 104, 20137-20141.

Pobric, G., Jefferies, E., \& Lambon Ralph, M. A. (2010). Amodal semantic representations depend on both anterior temporal lobes: Evidence from repetitive transcranial magnetic stimulation. Neuropsychologia. 48, 1336-1342.

Pobric, G., Lambon Ralph, M. A., \& Jefferies, E. (2009). The role of the anterior temporal lobes in the comprehension of concrete and abstract words: rTMS evidence. Cortex. 45, 1104-1110.

Postman, L., \& Keppel, G. (1970). Norms of word association. New York: Academic Press.

Rossi, S., Hallett, M., Rossini, P. M., \& Pascual-Leone, A. (2009). Safety, ethical considerations, and application guidelines for the use of transcranial magnetic stimulation in clinical practice and research. Clinical Neurophysiologv, 120, 2008-2039.

Saur, D., Kreher, B. W., Schnell, S., Kummerer, D., Kellmeyer, P., Vry, M. S., et al. (2008). Ventral and dorsal pathways for language. Proceedings of the National Academv of Sciences. U.S.A., 105, 18035-18040.

Schwartz, M. F., Kimberg, D. Y., Walker, G. M., Faseyitan, O., Brecher, A., Dell, G. S., et al. (2009). Anterior temporal involvement in semantic word retrieval: Voxel-based lesion-symptom mapping evidence from aphasia. Brain, 132, 3411-3427.

Snijders, T. M., Vosse, T., Kempen, G., Van Berkum, J. J., Petersson, K. M., \& Hagoort, P. (2009). Retrieval and unification of syntactic structure in sentence comprehension: An fMRI study using word-category ambiguity. Cerebral Cortex, 19, 1493-1503.

Snyder, H. R., Feigenson, K., \& Thompson-Schill, S. L. (2007). Prefrontal cortical response to conflict during semantic and phonological tasks. Journal of Cognitive Neuroscience. 19, 761-775.
Soni, M., Lambon Ralph, M. A., Noonan, K., Ehsan, S., Hodgson, C., \& Woollams, A. M. (2009). "L" is for tiger: Effects of phonological (mis)cueing on picture naming in semantic aphasia. Iournal of Neurolinguistics, 22, 538-547.

Thompson-Schill, S. L., D’Esposito, M., Aguirre, G. K., \& Farah, M. J. (1997). Role of left inferior prefrontal cortex in retrieval of semantic knowledge: A reevaluation. Proceedings of the National Academv of Sciences. U.S.A., 94, 14792-14797.

Thompson-Schill, S. L., Swick, D., Farah, M. J., D’Esposito, M., Kan, I. P., \& Knight, R. T. (1998). Verb generation in patients with focal frontal lesions: A neuropsychological test of neuroimaging findings. Proceedings of the National Academy of Sciences. U.S.A., 95, 15855-15860.

Wager, T. D., Jonides, J., \& Reading, S. (2004). Neuroimaging studies of shifting attention: A meta-analysis. Neuroimage. 22, 1679-1693.

Wagner, A. D., Pare-Blagoev, E. J., Clark, J., \& Poldrack, R. A. (2001). Recovering meaning: Left prefrontal cortex guides controlled semantic retrieval. Neuron, 31, 329-338.

Wallentin, M., Nielsen, A. H., Vuust, P., Dohn, A., Roepstorff, A., \& Lund, T. E. (2011). BOLD response to motion verbs in left posterior temporal gyrus during story comprehension. Brain and Language, 119, 222-225.

Walsh, V., \& Cowey, A. (2000). Transcranial magnetic stimulation and cognitive neuroscience. Natures Review Neuroscience, 1, 73-79.

Walsh, V., \& Rushworth, M. (1999). A primer of magnetic stimulation as a tool for neuropsychology. Neuropsychologia, 37, 125-135.

Wassermann, E. M. (1998). Risk and safety of repetitive transcranial magnetic stimulation: Report and suggested guidelines from the International Workshop on the Safety of Repetitive Transcranial Magnetic Stimulation, June 5-7, 1996. Electroencephalography and Clinical Neurophysiology, 108, 1-16.

Wassermann, E. M., Blaxton, T. A., Hoffman, E. A., Berry, C. D., Oletsky, H., Pascual-Leone, A., et al. (1999). Repetitive transcranial magnetic stimulation of the dominant hemisphere can disrupt visual naming in temporal lobe epilepsy patients. Neuropsychologia. 37, 537-544.

Whitney, C., Jefferies, E., \& Kircher, T. (2011). Heterogeneity of the left temporal lobe in semantic representation and control: Priming multiple versus single meanings of ambiguous words. Cerebral Cortex, 21, 831-844.

Zempleni, M. Z., Renken, R., Hoeks, J. C., Hoogduin, J. M., \& Stowe, L. A. (2007). Semantic ambiguity processing in sentence context: Evidence from event-related fMRI. Neuroimage, 34, 1270-1279. 